\title{
Using Discourse Predictions for Ambiguity Resolution
}

\author{
Yan Qu, Carolyn P. Rosé and Barbara Di Eugenio \\ Computational Linguistics Program \\ Department of Philosophy \\ Carnegie Mellon University \\ Pittsburgh, PA 15213 \\ $\{$ yqu,cprose $\} @ c s . c m u . e d u, d i e u g e n i @ c m u . e d u$
}

\begin{abstract}
In this paper we discuss how we apply discourse predictions along with non context-based predictions to the problem of parse disambiguation in Enthusiast, a Spanish-to-English translation system (Woszcyna et al., 1993; Suhm et al., 1994; Levin et al., 1995). We discuss extensions to our plan-based discourse processor in order to make this possible. We cvaluate those extensions and demonstrate the advantage of exploiting context-based predictions over a purely non context-based approach.
\end{abstract}

\section{Introduction}

$\Lambda$ system which processes spoken language must address all of the ambiguities arising when procossing written language, plus other ambiguities specific to the speech processing task. These include ambiguities derived from speech disfluencies, speech recognition errors, and the lack of clearly marked sentence boundaries. Because a large flexible grammar is necessary to handle these features of spoken language, as a side-effect the number of ambiguities increases. In this paper, we discuss how we apply discourse predictions along with non context-based predictions to the problem of parse disambiguation. 'This work has been carried out in the context of Iinthusiast, a Spanish-to-English speech-to-speech translation system (Woszcyna et al., 1993; Suhm et al., 1994; Levin et al., 1995), which currently translates spontancous dialogues between two people trying to schedule a meeting time.

A key feature of our approach is that it allows multiple hypotheses to be processed through the system in parallel, and uses context to disarnbiguate among alternatives in the final stage of the process, where knowledge can be exploited to the fullest extent. In our system, numerical predictions based on the more local utterance level are generated by the parser. 'The larger discourse context is processed and maintained by a plan-based discourse processor, which also produces contextbased predictions for ambiguities. Our goal was to combine the predictions from the context-based discourse processing approach with those from the non context-based parser approach.

In developing our discourse processor for disambiguation we needed to address three major issues. rirst, most plan-based or finite state automaton based discourse processors (Allen and Schubert, 1991; Smith, Hipp, and Biermann, 1995; Lambert, 1993; Reithinger and Maier, 1995), including the one we initially developed (Rosé et al., 1995), only take one semantic representation as input at a time: thus, we had to extend the discourse processor so that it can handle multiple hypotheses as input. Secondly, we needed to quantify the disambiguating predictions made by the plan-based discourse processor in order to combine these predictions with the non context-based ones. Finally, wo needed a method for combining context-based and non context-based predictions in such a way as to reflect not only which factors are important, but also to what extent they are important, and under what circumstances. We assume that knowledge from different sources provides different perspectives on the disambiguation task, each specializing in different types of ambiguities.

In this paper, we concentrate on the first two issues which are imperative to integrate a traditional plan-based discourse processor into the disambiguation module of a whole system. The third issue is very important for successful combination of predictions from different knowledge sources. We address this issue elsewhere in (Rosé and $Q \mathbf{u}$, 1995).

The paper is organized as follows: First, wo briefly introduce the Enthusiast speech translation system and discuss the ambiguity problem in Enthusiast. 'Then wo discuss our discourse processor, focusing on those characteristics needed to generatc predictions for disambiguation. Finally, we evaluate our performance, and demonstrate. that the use of discourse context improves performance on disambiguation tasks over a purely non context-based approach in the absence of $\mathrm{cu}^{-}$ mulative error. 


\section{System Description}

The main modules of our system include speech recognition, parsing, discourse processing, and generation. Processing begins with the speech input in the source language. The top best hypothesis of the speaker's utterance is then passed to the parser. The GLR* parser (Lavie, 1995) produces a set of interlingua texts, or Irits, for a given sentence. For robustiness, the GLR* parser can skip words in the inpul sentence in order to find a partial parse for a sentence which otherwise would not be parsable. An IIT' is a frame-based language independent meaning representation of a sentence. 'The main components of an II'T' are the spech act (e.g., suggest, accept, reject), the sentence type (c.g., state, query-if, fragment), and the main semantic frame (e.g., free, busy). An example of an ILT' is shown in Figure 1. 'l'he parser may produce many llís for a single sen tence, sometimes as many as one hundred or more.

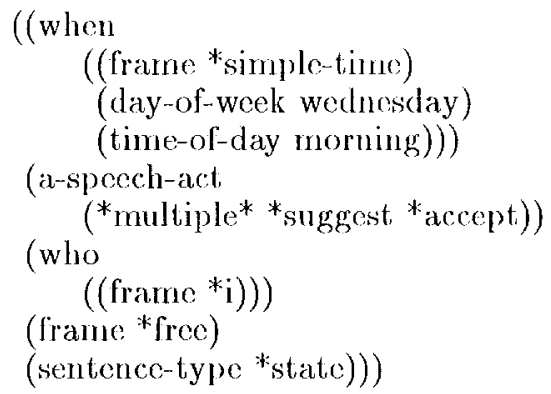

Sentenco: I could do it Wednesday morning too.

Figure 1: An Example ILT

The resulting set of IITS is then sent to the discourse processor. 'Ihe discourse processor, based on Lambert's work (Lambert and Carberry, 1992; Lambert, 1993), disambiguates the specch act of each sentence, nomalizes temporal expressions from context, and incorporates the sentence into the discourse context represented by a plan tree. The discourse processor also updates a calendar which keeps track of what the speakers have said about their schedules. We will discuss the dis course processor and how we extended it for the disambiguation task in Section 4.

\section{Ambiguity in Enthusiast}

Because the spontancous scheduling dialogues are unrestricted, ambiguity is a major problem in Linthusiast. We gauge aubiguitios in terms of differences between members of tho set of IL'Ts produced by the parser for the sanne source sentence. As wo mentioned earlier, the disanbiguation task bencfits from both non context-and context-hased methods. We observed that some classes of ambiguities can be more perspicuously dealt with in onc way or the other.

\subsection{Non Context-Based Disambiguation}

When the parser produces more than one II'L for a single sentence, it scores these ambiguities according to three different non context-based disambiguation methods. 'The first method, based on (Carroll and Briscoe, 1993), assigns probabilities to actions in the GL $R^{*}$ parser's parse table. 'The probabilities of the parse actions induce statistical scores on alternative parse trees, which are then used for parse disambiguation. 'I'he resulting score is called the statistical score. Tho second method the parser uses to score the II T': makes use of penalties manually assigned to different rules in the parsing grammar. The resulting score from this method is called the grammar preference score. 'The third score, called the parser score, is a heuristic combination of the previous two scores plus other information such as the number of words skipped. These three non context-based scores will be referred to later when we discuss combining non context-based predictions with context-based oncs.

Error analysis of parser disambiguation output shows that the CILR* parser handles well ambiguities which are not strongly dependent upon the: context for a rcasonable interpretation. For example, the Spanish word una can mean either one or $a$, as an indefinite reference. The parser always chooses the indefinite reference ineaning since the vast majority of training examples use this sense of the word. Moreover, since in this case incorrect disambiguation does not adversely affect transla. tion quality, it makes sense to handle this ambiguity in a purcly non context-based manner.

\subsection{Contcxt-Based Disambiguation}

While a broad range of ambiguitios can be han dled well in a non context-based manner, somo ambiguitics must be trated in a context sensitive manner in order to be translated correctly. Table 1 lists some examples of these types of arrt biguities. Wach type of ambiguity is catcgorized by comparing either diflerent slots in alternative IL'I's or different values in ambiguons IL'I slotis given the same input utterance.

For example, one type of ambignity best handled with a context-based approach is the day vs hour arnbiguity, exemplified by the phrase dos a cualro. It can moan either the second al four, the sccond to the fourth or two to four. Out of context, it is impossible to tell which is the best interpretation. Contextual information makes it possible to choose the correct interpretation. For example, if the speakers are trying to establish a date when they can moet, thon the second to the fourlh is the most likely interpretation. However, 


\begin{tabular}{|c|c|c|}
\hline Types of Ambiguity & Description & Examples \\
\hline day vs hour & $\begin{array}{l}\text { a temporal expression can be } \\
\text { recognized as a day or an hour }\end{array}$ & $\begin{array}{l}\text { dos a cuatro } \\
\text { second at four or } \\
\text { sccond to fourth or } \\
\text { two to four }\end{array}$ \\
\hline state vs query-if & $\begin{array}{l}\text { ambiguity between sentence } \\
\text { type state or query-if }\end{array}$ & $\begin{array}{l}\text { está bien } \\
\text { It's } O K \text { or } \\
\text { Is it } O K \text { ? }\end{array}$ \\
\hline speaker reference & $\begin{array}{l}\text { ambiguity between pro-drop } \\
\text { pronouns }\end{array}$ & $\begin{array}{l}\text { también podría ese dia } \\
\text { also i could that day or } \\
\text { also you could that day }\end{array}$ \\
\hline tense & $\begin{array}{l}\text { ambiguity between past tense } \\
\text { and present tense }\end{array}$ & $\begin{array}{l}\text { dónde nos encontramos } \\
\text { where are we meeting or } \\
\text { where were we meeting }\end{array}$ \\
\hline how vs greet & $\begin{array}{l}\text { ambiguity between frame how } \\
\text { and greet }\end{array}$ & $\begin{array}{l}\text { qué tal } \\
\text { How are you? or } \\
\text { How is that? }\end{array}$ \\
\hline when vs where & $\begin{array}{l}\text { anbiguity between when slot } \\
\text { and where slot }\end{array}$ & $\begin{array}{l}\text { sabado quince } \\
\text { Saturday the fifteenth or } \\
\text { Saturday building } 15\end{array}$ \\
\hline
\end{tabular}

Table 1: Examples of Context-Sensitive Ambiguities

if the speakers have already chosen a date and are negotiating the exact time of the meeting, then only the meaning two lo four makes sense.

Some sentence type ambiguities are also context-based. For example, L'stá bien can be either the statement $I t$ is good or the question Is it good?. This is an example of what we call the state vs query-if ambiguity: in Spanish, it is impossible to tell out of context, and without information about intonation, whether a sentence is a statement or a yes/no question. However, if the same spcaker has just made a suggestion, then it is more likely that the speaker is requesting a response from the other speaker by posing a question. In contrast, if the previous speaker has just made a suggestion, then it is more likcly that the current speaker is responding with an accepting statement than posing a question.

In general, we base our context-based predictions for disambiguation on turn-taking information, the stage of negotiation, and the speakers' calendar information. This information is encoded in a sct of context-based scores produced by the discourse processor for each ILT.

\section{Discourse Processing and}

\section{Disambiguation}

Context-based ranking of ambiguities is performed by the plan-based discourse processor described in (Rosé et al., 1995) which is based on (Lambert and Carberry, 1992; Lambert, 1993). Originally, our discourse processor took as its input the single best parse returned by the parser. The main task of the discourse processor was to relate that representation to the context, i.e., to the plan tree. In general, plan inference starts from the surface forms of sentences. Then speechacts are inferred. Multiple speech-acts can be inferred for one ILT. A separate inference chain is created for cach potential speech act performed by the associated IL'T. Preferences for picking one inference chain over another were determined by the focusing heuristics, which provide ordered expectations of discourse actions given the existing plan tree. Our focusing heuristics, described in detail in (Rosé et al., 1995), are an extension of those described in (Lambert, 1993). In determining how the inference chain attaches to the plan tree, the speech-act is recognized, since each inference chain is associated with a single speech-act.

As mentioned in the introduction, for a planbased discourse processor to deal with ambiguities, three issues need to be addressed:

1. The discourse processor must be able to deal with more than one semantic representation as input at a time. Note that simply extending the discourse processor to accept multiple ILTs is not the whole solution to the disambiguation problem: finer distinctions must be made in terms of coherence with the context in order to produce predictions detailed enough to distinguish between alternative ILTs.

2. Before context-based predictions can be combined with quantitative non context-based predictions, they must be quantified. It was necessary to add a mochanism to produce more detailed quantifiable predictions than those produced by the original focusing heuristics described in (Rosé et al., 1995).

3. Pinally, context-based predictions must be combined successfully with non-contextbased ones. 'The discourse processor must be able to weigh these various predictions in order to determine which ones to believe in specific circumstances.

Thus, we extended our original discourse processor as follows. It takes multiple arnbiguous IITs from the parser and computes three quantified discourse scores for each ambiguity. The discourse scores are derived by taking into account 
atlachment preferences to the discourse tree, as reflected by two kinds of focusing scores, and the score returned by the graded constrainls, a new type of constraint we introduced. Then for each ambiguity the discourse processor combines theso three kinds of context-based scores with the non context-based scores produced by other modules of the systern to make the final choice, and returus the chosen II'I'. As in the first version of the discourse processor, the chosen II'T is attached to the plan tree and a speech act is assigned to it. We discuss now how the discourse scores are derived. Note that lower values for all scores are preferred.

\section{$4.1 \quad$ Focusing scores}

'The focusing scores are derived from focusing heuristics based on (Sidner, 1981; Lambert, 1993; Rosé et al., 1995). 'The focusing heuristics identify the most coherent relationship between a new inference chain and the discourse plan tree. Attachment preferences by the focusing heuristics are translated into numerical preference scores based on attachment positions and the length of the inference chains. 'The assignment of focusing scores reflects the assumption that the most coherent move in a dialogue is to continue the most salient focused actions, namely, the ones on the rightmost froution of the plan tree. The first focusing score is a boolean focusing flag. It returns 0 if the inference chain for the associated IL'T attaches to the rightmost frontior of the plan tree, 1 if it cither attaches to the tree but not to the right frontier or doesn't attach to the tree. The second focusing score, the focusing score proper, assigns a score between 0 and 1 indicating how far up the rightmost frontier the inference chain attaches. The maximal score is assigned in the case that the inference chain does not attach.

\subsection{Graded constraints}

Once the discourse processor was extended to accept multiple ILI's as input, it became clear that for most ambignous parses the original focusing heuristics did not provide enough information to distinguish among the alternatives. Our solution was to modify the discourse processor's constraint processing mochanism, making it possible to bring more domain knowledge to bear on the disambiguation task. In the original discourse processor, all of the constraints on plan operators, which we call climination constraints, were used solely for the purpose of binding variables and eliminating certain inference possibilities. Their purpose was to climinate provably wrong inferences, and in this way to give the focusing houristics a higher likelihood of selecting the correct inference chain from the remaining set.

We introduced a different type of constraint, graded constraints, inspired by the concept of graded unification discussed in (Kim, 1994). Un- like elimination constraints, they neither bind variables nor eliminate any inferencos. Graded constraints always roturn tirue, so they cannot climinate inferences. Ilowever, they assign numerical penalties or preferences to inference chains based on domain specific information. T'his information is then used to rank the set of possible inferences left after the elimination constraints are processed.

for example, consider the day versus hour arribiguity we discussed carlicr. In most cases inference chains for [IT's with this ambiguity have the same focusing scores. We introduce the possible-time constraint to check whether the temporal constraints conflict with the dynamic calendar or the recorded dialogue date when the inference chains are built. If the temporal information represented in an II'T is in conflict with the dialogue record date (e.g., scheduling a time before the record date) or with the temporal constraints already in the calendar (e.g., propose a time that is already rejected), a penalty score is assigned to that isference chain; otherwise, a default value (i.e. no penalty) is returned. Several graded constraints may be fired in one inference chain. Penalties or preferences for all graded constraints in the inference chain are summed together. 'The result is the graded constraint score for that ambiguity.

Introducing gruded constraints has two advan. tages over adding more climination constraints. As far as the system in general is concerned, graded constraints only give preferences, they do not rule out inferencing and attachment possibilities: thus, introducing new constraints will not danrage the broad coverage of the system. As far as the discourse processor is concerned, it would be possible to achieve the same effect by adding tuore climination constraints, but this would make it necessary to intioduce moro fine-tuned plan opcrators geared towards specilic cases. By introducing graded constraints wo avoid expanding the scarch space among the plan opcrators.

\subsection{Combining Predictions}

Once the information from the graded constraints and the focusing scoros is available, the challonging problem of combining these context-based predictions with the non context-based ones arises. We experimented with two methods of automatically lcarning functions for combining our six scores into one composite score, namely a genetic programming approach and a neural net approach. 'The basic assumption of our disambiguation approach is that the context-based and non context-based scores provide different perspectives on the disambiguation task. T'hey act together, each specializing in different types of cases, to constrain the final result. Thus, we want our learning approach to learn not only which factors are important, but also to what extent they are 
important, and under what circumstances. The genetic programming and neural net approaches are ideal in this respect.

Genetic programming (Koza, 1992; Koza, 1994) is a method for "evolving" a program to accomplish a particular task, in this case a function for computing a composite score. This technique can learn functions which are efficient and humanly understandable and editable. Moreover, because this technique samples diffcrent parts of the search space in parallel, it avoids to some extent the problem of selecting locally optimal solutions which are not globally optimal.

Connectionist approaches have been widely used for spoken language processing and other areas of computational linguistics, e.g., (Wermpter, 1994; Miikkulainen, 1993) to narne only a few. Connectionist approaches are able to learn the structure inherent in the input data, to make fine distinctions between input patterns in the presence of noise, and to integrate different information sources.

We refer the reader to (Rosé and Qu, 1995) for full details about the motivations underlying the choice of these two methods as well as the advantages and disadvantages of each.

\section{Evaluation}

Both combination methods, the genetic programming approach and the neural net approach, were trained on a set of 15 Spanish scheduling dialogues. Thcy were both tested on a set of five previously unseen dialogues. Only sentences with multiple ILT's, at least one of which was correct, were used as training and testing data. Altogether 115 sentences were used for training and 76 for lesting.

Wo evaluated the performance of our two methods by comparing them to two non context-based ones: a baseline method of selecting a parse randomly, and a Statistical Parse Disambiguation method. The Statistical Parse Disambiguation method makes use of the three non context-based scores described in Section 3. The two contextbased approaches combine the three non contextbased scores as well as the three context-based scores, namely the focusing flag, the focusing score, and the graded constraint score.

Table 2 reports the percentages of ambiguous sentences correctly disambiguated by each method. We present two types of performance statistics on the testing set: without cumulative error Testing without $C E$ and with cumulative error Testing with CE. Cumulative error builds up when an incorrect hypothesis is chosen and incorporated into the discourse context, causing future predictions based on discourse context to be inaccurate. Notice that for the two non contextbased approaches, the performance figures for both kinds of testing are the same becausc cumulative error is only an issue for context-based approaches.

Our results show that the discourse processor is indeed making useful predictions for disambiguation: when we abstract away the problem of cumulative error, we can achieve an improvement, of $13 \%$ with the genetic programming approach and of $2.5 \%$ with the neural net approach over the parser's non-context based statistical disambiguation technique. For example, we were able to achieve almost perfect performance on the state vs query-if ambiguity, missing only one case with the genetic programming approach; thus, for this ambiguity, we can trust the discourse processor's prediction.

However, our results also indicate that we have not solved the whole problem of combining non context- and context-based predictions for disambiguation. In the face of cumulative error, both of the two discourse combination approaches suffer from performance degradation, though to a different extent. Our current direction is to scek a solution to the cumulative error problem. Some preliminary results in this regard are discussed in (Qu et al., 1996).

\section{Conclusions}

In this article we have discussed how we apply predictions from our plan-based discourse processor to the problem of disambiguation. Our evaluation demonstrates the advantage of incorporating context-based predictions into a purely non context-based approach. While our results indicate that we have not solved the whole problem of combining non context- and context-based predictions for disambiguation, they show that the discourse processor is making useful predictions and that we have combined this information successfully with the non context-based predictors.

Our current efforts are aimed at solving the cumulative error problem in using discourse context. We noticed that cumulative error is especially a problem in spontaneous speech systems where unexpected input, disfluencies, out-of-domain sentences and missing information cause the deterioration of the quality of context. One possibility is to reassess and reestablish the context state when a conflict is detected between context and other predictions. A second proposal is to keep the nbest hypotheses and to choose one only after having processed a sequence of inputs. Preliminary experiments show that both proposals help reduce the adverse effect of the cumulative error problem.

Our results also suggest another possible avenue of future development. Instead of trying to learn a general function for combining various information sources, we could decide which source of information to trust in a particular case and classify 


\begin{tabular}{|l|c|c|c|}
\hline & Training & Testing without CE & Testing with CE \\
\hline Randona & $32 \%$ & $45 \%$ & $45 \%$ \\
\hline Statistical Parse Disanbiguation & $76.5 \%$ & $76.3 \%$ & $76.3 \%$ \\
\hline DP Genetic Progranming & $91.6 \%$ & $89.5 \%$ & $60 \%$ \\
\hline DP Nenral Net & $85.2 \%$ & $78.8 \%$ & $71.3 \%$ \\
\hline
\end{tabular}

'lable 2: Disambiguation of All Ambiguous Sentences

the type of ambiguity at hand with the best approach for this ambiguity. 'I'his could be accomplished, for example, with a decision trec learning approach.

\section{Acknowledgements}

The authors would like to thank Lori Levin, Alon lavie and $\Lambda$ lex Waibel for comments on the work reported here and thank the two anonymous reviewers for comments on the earlier version of the paper. The work is supported in part by a grant. from the Department of Defiense.

\section{References}

Allen, J. F. and L. K. Schubert. 1991. The Trains Project. Ph.I). thesis, University of Rochester, School of Computer Science.

Garroll, J. and 'T. Briscoe. 1993. Generalizod probabilistic I,R parsing of natural language (corpora) with unificalion-based granmars. Computalional linguistics, 19(1).

Sidner, (:. I. 1981. Focusing for Lnterpretation of l'ronouns. American Journal of Compulational Linguistics, 7(4):217 231 .

Kim, A. 1994. Graded unification: A framework for interactive processing. In Proceedings of the Associalion for Computational Linguistics.

Koza, J. 1992. Genetic Programming: On the Programming of Compulers by Means of Natural Selection. MI'I' P'ress.

Koza, J. 1994. Genelic Programming II. MIT Press.

Lambert, I. 1993. Recognizing Complex Discourse Acls: A Triparlile Plan-Based Model of Dialogue. Ph.D. thesis, Department of Computer Science, University of Delaware.

Lambert, L. and S. Carberry. 1992. Modeling negotiation subdialogues. In Procedings of the ACl.

Lavie, A. 1995. A Crammar Based Robust Parser I'or Spontaneous Speech. Ph.D. thesis, School of Computer Science, Carnogie Mellon University.

Levin, I., O. Glickman, Y. Qu, D. Gates, A. Lavie, C. P. Rosé, ( Van Lss-Dykema, and
A. Waibel. 1995. Using context in machine translation of spoken language. In Theoretical and Melhodological Issues in Machine Translation.

Miikkulainen, R. 1993. Subsymbolic Nalural Language Processing: An Integraled Model of Scripts, Lexicon, and Memory. The MrT Press.

Qu, Y., B. Di Lugenio, A. Lavie, I. S. Levin, and C. P’. Rosć. 1996. Minimizing Cumulative Error in Discourse ('ontext. 'To appear in $\mathrm{LCAl}$ Workshop Proceedings on Dialogue Processing in Spoken Language Systems.

Reithinger, N. and Vi. Maier. 1995. Utilizing sta listical dialoguc act processing in Verbmobil. In Proceedings of the ACl.

Rose, C. P., B. Di Dugento, L. S. Levin, and (. Van Iss-1)ykema. 1995. 1)iscourse processing of dialogues with multiple threads. In Prorecdings of the $A C L$.

Rosé, (.. P. and Y. Qu. 1995. Automatically learning lo Use Discourse Information for Disambiguation. Center for Machine Iranslation, Carnegic Mellon University. 'Technical Report.

Smith, R. W., D. R. Hipp, and $\Lambda$. W. Biemmann. 1995. An architecture for voice dialogue systerns based on prolog-style theorem proving. Computational Linguistics, 21(3):218-320.

Suhm, B., L. Levin, N. Coccaro, J. Carbonell, K. Horiguchi, R. Isotani, A. Lavic, L. Mayfield, (. P. Rosé, (C. Van Fiss Lykema, and A. Waibel. 1994. Speech-language integration in a multilingual speech translation system. In Proceedings of the $A A A I$ Workshop on Integration of Natural Language and Speech Processing.

Wermpter, S. 1994. Connectionist learning of flat syntactic analysis for specch/language systems. In Proceedings of the International Conference on Artificial Neural Networks.

Woszcyna, M., N. Coccaro, A. Eisele, A. Lavie, A. McNair, 'I'. Polzin, I. Rogina, C. P'. Rosé, T'. Sloboda, M. 'Tomita, J. 'Isutsumi, N. Waibel, A. Waibel, and W. Ward. 1993. Recent advances in JANUS: a speech translation system. In Proceedings of the ARPA IIuman Languages Technology Workshop. 